\title{
Terahertz response of acoustically driven optical phonons
}

\author{
R. H. Poolman, E. A. Muljarov, and A. L. Ivanov \\ Department of Physics and Astronomy, Cardiff University, Cardiff CF24 3AA, United Kingdom
}

(Received 8 April 2010; revised manuscript received 24 May 2010; published 17 June 2010)

\begin{abstract}
The manipulation of transverse-optical (TO)-phonon polaritons and the associated terahertz (THz) light field by means of an ultrasound acoustic wave is proposed and illustrated by calculating the TO-phonon-mediated $\mathrm{THz}$ response of acoustically pumped $\mathrm{CuCl}$ and $\mathrm{TlCl}$ crystals. We show the high-contrast acoustically induced change in the $\mathrm{THz}$ reflectivity and multiple $\mathrm{THz}$ Bragg replicas, which are associated with the far-infraredactive TO-phonon resonance driven by the ultrasonic wave. The effect, which stems from phonon anharmonicity and deals with the resonantly enhanced acousto-optical susceptibilities, refers to an operating acoustic intensity $I_{\mathrm{ac}} \sim 1-100 \mathrm{~kW} / \mathrm{cm}^{2}$ and frequency $\nu_{\mathrm{ac}} \sim 0.1-1 \mathrm{GHz}$. Due to the anomalously small interaction length between the acoustic and optical fields, possible applications of the effect are in $\mathrm{THz}$ spectroscopy and $\mathrm{THz}$ acousto-optic devices.
\end{abstract}

DOI: $10.1103 /$ PhysRevB.81.245208

PACS number(s): 71.36. $+\mathrm{c}, 43.35 .+\mathrm{d}$

\section{INTRODUCTION}

Since the pioneering work by Kun Huang, ${ }^{1}$ the physics of infrared polaritons associated with transverse-optical (TO) phonons has emerged as a well-established discipline. Recently, room-temperature polaritonics was implemented for processing and coherent control of the terahertz $(\mathrm{THz})$ light field. ${ }^{2,3}$ In addition to conventional infrared spectroscopy and continuous-wave (cw) Raman-scattering experiments, broadband $\mathrm{THz}$ time-domain spectroscopy has been developed and successfully applied to characterize picosecond (ps) and sub-ps dynamics of infrared-active TO phonons ${ }^{4}$ and to visualize the polariton dispersion associated with these vibrational modes. ${ }^{5}$ Furthermore, the THz polariton spectra allow the study of unusual lattice dynamics, e.g., in ferroelectrics ${ }^{6}$ and negative thermal-expansion compounds. ${ }^{7,8}$ However, the spectrally resolved control of the electromagnetic field associated with far-infrared polaritons still remains a very challenging task of $\mathrm{THz}$ optics.

In this paper, we propose an acoustic modulation of TOphonon polaritons in order to drastically change their optical response in the THz band. Phonon anharmonicity, which can be large in some dielectric and semiconductor materials and particularly strong for soft TO-phonon modes, leads to the coupling between a coherent (pumping) acoustic wave (AW) and infrared-active TO phonons. In this case one deals with an acoustically induced Autler-Townes effect, which gives rise to spectral gaps $\Delta_{N}$ in the $\mathrm{THz}$ polariton spectrum of AW-driven TO phonons, and is akin to the acoustical Stark effect for optically allowed excitons. ${ }^{9-14}$ The AW-induced gaps $\Delta_{N}$, which open up in the polariton spectrum and develop with increasing acoustic intensity $I_{\mathrm{ac}}$, are due to the $N$ th-order resonant acoustic-phonon transitions within the polariton dispersion branches. These forbidden-energy gaps strongly modify the optical response of TO-phonon polaritons and make possible the effective AW-controlled manipulation of the $\mathrm{THz}$ field.

The spectrally resolved AW control of the $\mathrm{THz}$ field propagation can also be interpreted in terms of Bragg diffraction of far-infrared polaritons by the pumping AW: we analyze the use of phonon anharmonicity to create an acousti- cally induced Bragg grating. In this case the contrast of the AW grating is dictated by the efficiency of the scattering channel "TO phonon \pm acoustic phonon (two acoustic phonons) $\leftrightarrow$ TO phonon" for cubic (quartic) phonon anharmonicity. Thus the scattering of $\mathrm{THz}$ light is mediated and strongly enhanced by the TO-phonon resonance. This results in large acousto-optical nonlinearities and therefore in an anomalously short interaction length $\ell_{\text {int }}$ between the acoustic and optical fields needed for the formation of the Bragg replicas.

Possible applications of governing far-infrared polaritons by using an ultrasonic acoustic field include frequencytunable $\mathrm{THz}$ detectors and filters, Bragg switchers, and frequency converters. The size of these devices would be much smaller than conventional acousto-optic equivalents, due to the rather small operating length scale, $\ell_{\text {int }} \sim 100 \mu \mathrm{m}$.

In Sec. II, we discuss a model for far-infrared polaritons parametrically driven by the ultrasonic acoustic field, derive the macroscopic equations for resonantly coupled $\mathrm{THz}$ light field and polarization associated with TO phonons, and finally calculate the total Bragg reflectivity of TO-phonon polaritons. In Sec. III, the AW-induced response of THz polaritons in bulk $\mathrm{CuCl}$ (cubic phonon anharmonicity) and $\mathrm{TlCl}$ (quartic phonon anharmonicity) is modeled to illustrate the high efficiency of the effect and its potential for device applications. In Sec. IV, we discuss the results. A short summary of the work is given in Sec. V.

\section{MODEL}

The Hamiltonian of far-infrared polaritons coherently driven by a cw bulk acoustic wave $\left\{\mathbf{k}, \omega_{\mathrm{ac}}(k)\right\}$ is given by

$$
\begin{aligned}
H= & H_{0}+\hbar \sum_{\mathbf{p}}\left[2 \widetilde{m}_{4} I_{\mathrm{ac}} b_{\mathbf{p}}^{\dagger} b_{\mathbf{p}}+\left(m_{3} I_{\mathrm{ac}}^{1 / 2} e^{-i \omega_{\mathrm{ac}} t} b_{\mathbf{p}}^{\dagger} b_{\mathbf{p}-\mathbf{k}}+\text { H.c. }\right)\right. \\
& \left.+\left(m_{4} I_{\mathrm{ac}} e^{-2 i \omega_{\mathrm{ac}} t} b_{\mathbf{p}}^{\dagger} b_{\mathbf{p}-2 \mathbf{k}}+\text { H.c. }\right)\right]
\end{aligned}
$$

with $H_{0}$ the conventional polariton Hamiltonian of infraredactive TO phonons, ${ }^{15} b_{\mathbf{p}}$ the TO-phonon operator, $\omega_{\mathrm{ac}}$ $=2 \pi v_{\mathrm{ac}}=v_{\mathrm{s}} k, v_{\mathrm{s}}$ the sound velocity, and $m_{3}\left(m_{4}\right.$ and $\left.\widetilde{m}_{4}\right)$ the matrix element associated with cubic (quartic) anharmonic- 
ity. The macroscopic equations, which describe the control of $\mathrm{THz}$ polaritons by applying the acoustic field of an arbitrary profile, $I_{\mathrm{ac}}=I_{\mathrm{ac}}(\mathbf{r}, t)$, are

$$
\begin{aligned}
& {\left[\frac{\varepsilon_{\mathrm{b}}}{c^{2}} \frac{\partial^{2}}{\partial t^{2}}-\nabla^{2}\right] \mathbf{E}(\mathbf{r}, t)=-\frac{4 \pi}{c^{2}} \frac{\partial^{2}}{\partial t^{2}} \mathbf{P}(\mathbf{r}, t),} \\
& {\left[\frac{\partial^{2}}{\partial t^{2}}+2 \gamma_{\mathrm{TO}} \frac{\partial}{\partial t}+\Omega_{\mathrm{TO}}^{2}+4 \Omega_{\mathrm{TO}} \widetilde{m}_{4} I_{\mathrm{ac}}(\mathbf{r}, t)\right.} \\
& +4 \Omega_{\mathrm{TO}} m_{3} I_{\mathrm{ac}}^{1 / 2}(\mathbf{r}, t) \cos \left(\omega_{\mathrm{ac}} t-\mathbf{k r}\right) \\
& \left.+4 \Omega_{\mathrm{TO}} m_{4} I_{\mathrm{ac}}(\mathbf{r}, t) \cos \left(2 \omega_{\mathrm{ac}} t-2 \mathbf{k r}\right)\right] \mathbf{P}(\mathbf{r}, t) \\
& =\frac{\varepsilon_{\mathrm{b}}}{4 \pi} \Omega_{\mathrm{R}}^{2} \mathbf{E}(\mathbf{r}, t),
\end{aligned}
$$

where $\mathbf{E}$ and $\mathbf{P}$ stand for the light field and TO-phonon polarization, respectively, $\Omega_{\mathrm{TO}}$ is the TO-phonon frequency, $\Omega_{\mathrm{R}}$ is the polariton Rabi frequency, $\varepsilon_{\mathrm{b}}$ is the background dielectric constant in the infrared, and $\gamma_{\mathrm{TO}}$ is the damping rate of TO phonons, mainly due to their decay into shortwavelength acoustic phonons. Equations (2) and (3) refer to simple cubic lattices with spatially isotropic long-wavelength anharmonicity and optical response. For cw acoustic excitations, when $I_{\mathrm{ac}}(\mathbf{r}, t)=I_{\mathrm{ac}}=$ const, Eqs. (2) and (3) yield the same quasienergy spectrum as that of the quadratic Hamiltonian (1). If $I_{\mathrm{ac}}=0$, Eqs. (2) and (3) reduce to the standard TO-phonon polariton equations. ${ }^{1}$ The fourth term on the lefthand side (lhs) of the polarization Eq. (3) yields a Stark shift of the TO-phonon frequency, which is $\propto I_{\mathrm{ac}}$ and is associated with the quartic phonon nonlinearity. The last two terms on the lhs of Eq. (3) give rise to the Bragg spectrum of TOphonon polaritons driven by the AW.

In Eqs. (1) and (3), the matrix elements $m_{3,4}$ and $\widetilde{m}_{4}$ are given by

$$
\begin{gathered}
m_{3}=6\left(\frac{v_{0}}{\hbar^{3} v_{\mathrm{s}}^{2} k}\right)^{1 / 2} V_{3}(\mathbf{k}, \mathbf{p}-\mathbf{k},-\mathbf{p}) \\
m_{4}=12\left(\frac{v_{0}}{\hbar^{2} v_{\mathrm{s}}^{2} k}\right)\left[V_{4}(\mathbf{k}, \mathbf{k}, \mathbf{p}-2 \mathbf{k},-\mathbf{p})+V_{4}(\mathbf{k}, \mathbf{k},-\mathbf{p}, \mathbf{p}-2 \mathbf{k})\right] \\
\tilde{m}_{4}=24\left(\frac{v_{0}}{\hbar^{2} v_{\mathrm{s}}^{2} k}\right) V_{4}(\mathbf{k},-\mathbf{k}, \mathbf{p},-\mathbf{p})
\end{gathered}
$$

where $v_{0}$ is a volume of the primitive cell and

$$
\begin{aligned}
& V_{3}(\mathbf{k}, \mathbf{p}-\mathbf{k},-\mathbf{p})=\frac{1}{6}\left(\frac{\hbar^{3}}{8 v_{\mathrm{s}} k \Omega_{\mathrm{TO}}^{2}}\right)^{1 / 2} \Phi^{(3)}(\mathbf{k}, \mathbf{p}-\mathbf{k},-\mathbf{p}), \\
& V_{4}(\mathbf{k},-\mathbf{k}, \mathbf{p},-\mathbf{p})=\frac{1}{24}\left(\frac{\hbar^{2}}{4 v_{\mathrm{s}} k \Omega_{\mathrm{TO}}}\right) \Phi^{(4)}(\mathbf{k},-\mathbf{k}, \mathbf{p},-\mathbf{p}) .
\end{aligned}
$$

Here, $\Phi^{(3)}\left(\Phi^{(4)}\right)$ is proportional to the Fourier transform of the third-order (fourth-order) spatial derivative of the interatomic potential. The explicit formulas for $\Phi^{(3,4)}$ are given, e.g., in Refs. 16 and 17.

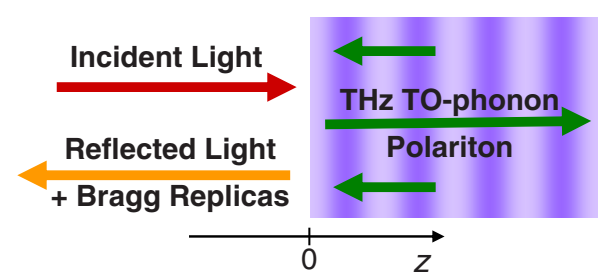

FIG. 1. (Color online) Schematic of optical excitation and backward Bragg scattering of an acoustically driven $\mathrm{THz}$ polariton. Vertical stripes symbolize the propagating bulk acoustic wave.

In the long-wavelength limit $k, p \ll 1 / a_{0}\left(a_{0}\right.$ is the lattice constant), relevant to the optics of TO-phonon polaritons, $\Phi^{(3,4)}$ are well approximated by $\Phi^{(3)}=\left(k a_{0}\right) C_{3}$ and $\Phi^{(4)}$ $=\left(k a_{0}\right)^{2} C_{4} \cdot{ }^{18}$ The anharmonicity constants $C_{3,4}$ can either be calculated by using modern $a b$ initio methods ${ }^{19,20}$ or evaluated from experimental data available for some anharmonic crystals. ${ }^{21-25}$ The above approximation of $\Phi^{(3,4)}$ also leads to the $k$-independent matrix elements $m_{3}$ and $\widetilde{m}_{4}=m_{4}$ in Eqs. (1) and (3).

Recently, the third-order coupling constant $V_{3}$ was inferred for some zinc-blende-type semiconductors (GaP, $\mathrm{CuCl}, \mathrm{CuBr}$, and $\beta$ - $\mathrm{ZnS}$ ) which exhibit strong and dominant cubic anharmonicity. ${ }^{21-24}$ The used experimental data refers to the decay of a long-wavelength TO phonon into shortwavelength longitudinal-acoustic and transverse-acoustic (TA) phonons. To adapt the inferred values of $V_{3}$ to $m_{3}$ in Eqs. (1)-(3), we use the Leibfried-Ludwig approximation ${ }^{26}$ according to which $\Phi^{(3)}$ in Eq. (5) is the same for the whole Brillouin zone. The parameter $m_{3} I_{\mathrm{ac}}^{1 / 2}$, which controls cubicanharmonicity-mediated manipulation of $\mathrm{THz}$ polaritons by means of a bulk TA wave, is evaluated for $\mathrm{CuCl}$ as $\hbar m_{3} I_{\mathrm{ac}}^{1 / 2}$ $\simeq 0.26 \mathrm{meV}(63 \mathrm{GHz})$ for $I_{\mathrm{ac}}=1 \mathrm{~kW} / \mathrm{cm}^{2}$. In thallium halides $(\mathrm{TlCl}$ and $\mathrm{TlBr}$ ), quartic anharmonicity is dominant with positive values of $V_{4}$. With the Leibfried-Ludwig approximation of Eq. (6), one can evaluate the control parameter $m_{4} I_{\mathrm{ac}}$ from the available experimental data on the real part of the TO-phonon self-energy. ${ }^{25} \mathrm{For} \mathrm{TlCl}$ pumped by a bulk TA wave we get $\hbar m_{4} I_{\mathrm{ac}} \simeq 0.4 \mathrm{meV}(97 \mathrm{GHz})$ for $I_{\mathrm{ac}}$ $=100 \mathrm{~kW} / \mathrm{cm}^{2}$.

We examine the optical response of an acoustically driven TO-phonon polariton in a one-dimensional geometry, when a semiconductor occupies the half space $z>0$, and a normally incident light field of frequency $\omega$ induces a $\mathrm{THz}$ polariton propagating collinearly to the pumping AW (see Fig. 1). In this case, apart from reflectivity at the same frequency $\omega$, down-converted Bragg replicas at $\omega+n \omega_{\mathrm{ac}}(n=-1,-2, \ldots)$ arise in the reflection spectrum, due to acoustically induced backward scattering of the polariton. In order to calculate the multiple Bragg replicas, we develop an approach more advanced than that used in conventional acousto-optics. For the latter, the acousto-optical susceptibilities are so weak that usually only one Bragg replica $n=+1$ or -1 is seen. In contrast, the TO-phonon resonance mediates and considerably enhances the coupling between the optical and acoustic fields, so that generally one has to take into account the whole series of the Bragg replicas and multiphonon transitions, thus treating the problem nonperturbatively.

The quasienergy polariton spectrum $\omega=\omega(p)$, calculated for real-valued $p$ (quasiparticle solution) by solving Eqs. (2) 


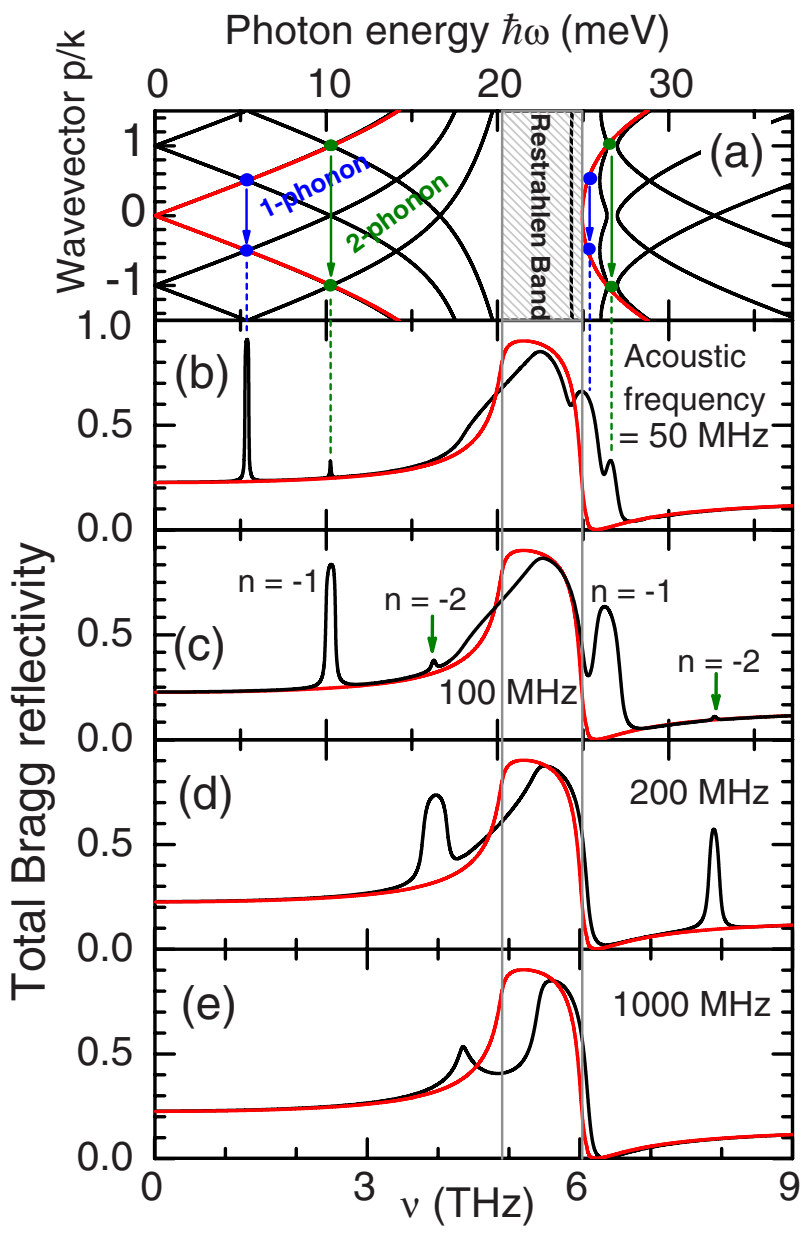

FIG. 2. (Color online) (a) The dispersion $\omega=\omega(p)$ of THz polaritons in $\mathrm{CuCl}$ driven by the bulk TA wave of $\nu_{\mathrm{ac}}=50 \mathrm{MHz}$, black lines, and the bare polariton spectrum, red (dark gray) lines. The wave vector $p$ is normalized to the acoustic wave vector $k$. The arrows highlight the $N$-TA-phonon resonant coupling between the polariton states $(N=1,2)$. [(b)-(e)] The calculated total Bragg reflectivity (black lines), $R=\Sigma_{n}\left|r_{n}\right|^{2}$, against the light frequency $\nu$ $=\omega /(2 \pi)$ for $\nu_{\mathrm{ac}}=50 \mathrm{MHz}, 100 \mathrm{MHz}, 200 \mathrm{MHz}$, and $1 \mathrm{GHz}$, respectively. The bare polariton reflectivity is shown by the red (dark gray) lines. The Bragg signals labeled in (c) as $n=-1$ and $n=-2$ are mainly due to $\left|r_{-1}\right|^{2}$ and $\left|r_{-2}\right|^{2}$, respectively. The acoustic intensity $I_{\mathrm{ac}}=25 \mathrm{~kW} / \mathrm{cm}^{2}$.

and (3) with $m_{4}=0$, is plotted in Fig. 2(a) for $\mathrm{CuCl}$ parametrically driven by the TA wave of frequency $\nu_{\mathrm{ac}}$ $=50 \mathrm{MHz}$ and constant intensity $I_{\mathrm{ac}}=25 \mathrm{~kW} / \mathrm{cm}^{2}$. The spectrum, which arises from spatial and temporal modulation of the crystal lattice, can be interpreted in terms of the Brillouin-zone picture, with acoustically induced energy gaps $\Delta_{N} \propto I_{\mathrm{ac}}^{N / 2}$ due to the $N$-phonon resonant transitions within the polariton dispersion branches. The spectral positions of the transitions are indicated in Fig. 2(a) by the vertical arrows. For the frequency scale used in Fig. 2(a), only the gaps $\Delta_{N=1}$ and $\Delta_{N=2}$ in the upper polariton branch are clearly seen.

From the air side, $z<0$ (see Fig. 1), the light field is given by
TABLE I. Parameters of bulk $\mathrm{CuCl}$ and $\mathrm{TlCl}$ used in the numerical calculations.

\begin{tabular}{lcc}
\hline \hline & $\mathrm{CuCl}$ & $\mathrm{TlCl}$ \\
\hline$\hbar \Omega_{\mathrm{TO}}(\mathrm{meV})$ & 20.28 & 7.81 \\
$\hbar \Omega_{\mathrm{R}}(\mathrm{meV})$ & 14.53 & 17.97 \\
$\hbar \gamma_{\mathrm{TO}}(\mathrm{meV})$ & 0.20 & 0.92 \\
$v_{\mathrm{S}}\left(10^{5} \mathrm{~cm} / \mathrm{s}\right)$ & 2.02 & 2.19 \\
$\hbar m_{3}\left(10^{-12} \mathrm{meV}^{1 / 2} \mathrm{~cm} \mathrm{~s}^{1 / 2}\right)$ & 0.10 & 0 \\
$\hbar m_{4}\left(10^{-27} \mathrm{~cm}^{2} \mathrm{~s}\right)$ & 0 & 0.64 \\
\hline \hline
\end{tabular}

$$
E(z<0, t)=e^{i q_{0} z} e^{-i \omega t}+\sum_{n} r_{n} e^{-i q_{n} z} e^{-i\left(\omega+n \omega_{\mathrm{ac}}\right) t}
$$

where $q_{n}=\left(\omega+n \omega_{\mathrm{ac}}\right) / c$ with $-n_{\max } \leq n \leq n_{\max }$ (we proceed up to $n_{\max }=60$ ) and $r_{n}$ stands for the amplitude of the outgoing Bragg replica $n$ normalized to the unity amplitude of the incoming light wave. The electric field propagating in the crystal $(z>0)$ is

$$
E(z>0, t)=\sum_{n, j} A_{j} E_{n j} e^{i\left(p_{j}+n k\right) z-i\left(\omega+n \omega_{\mathrm{ac}}\right) t} .
$$

Here, $p_{j}=p_{j}(\omega)$ is the wave vector associated with the quasienergy dispersion branch $j\left(-n_{\max } \leq j \leq n_{\max }\right)$ of the acoustically driven polariton, $E_{n j}$ are the corresponding normalized eigenvectors, and $A_{j}$ are the eigenmode amplitudes. Both $p_{j}=p_{j}(\omega)$ and $E_{n j}$ are the forced-harmonic solutions of Eqs. (2) and (3) for real-valued frequency $\omega$. The exponential on the right-hand side of Eq. (8) as well as the basic relationships $p_{j+s}(\omega)=p_{j}\left(\omega-s \omega_{\mathrm{ac}}\right)+s k$ and $E_{n, j+s}(\omega)=E_{n+s, j}(\omega$ $\left.-s \omega_{\mathrm{ac}}\right)$, with integer $s$, reflect the acoustic wave vector and frequency translational invariance of the quasienergy spectrum. The Maxwellian boundary conditions at $z=0$ together with Eqs. (7) and (8) yield,

$$
\delta_{n, 0}+r_{n}=\sum_{j} A_{j} E_{n j}, \quad \delta_{n, 0}-r_{n}=\sum_{j} A_{j} E_{n j} \frac{p_{j}+n k}{q_{n}} .
$$

The above set of $2\left(2 n_{\max }+1\right)$ linear equations determines $r_{n}$ and $A_{j}$.

\section{NUMERICAL MODELING}

In this section, by solving numerically Eqs. (2), (3), and (9), we model the terahertz response of acoustically pumped copper chloride and thallium chloride crystals. For the first (second) compound, we set $m_{4}=\tilde{m}_{4}=0 \quad\left(m_{3}=0\right)$ in Eq. (3), according to the relative strength of the cubic and quartic anharmonicity coefficients in $\mathrm{CuCl}(\mathrm{TlCl})$. Table I summarizes values of the parameters used in numerical simulations.

\section{A. Acoustically driven $\mathrm{THz}$ polaritons in bulk $\mathrm{CuCl}$}

In Figs. 2(b)-2(e), we compare the calculated total Bragg reflectivity, $R=R(\omega)=\Sigma_{n}\left|r_{n}\right|^{2}$ (black solid lines), with the reflectivity of the acoustically unperturbed $\mathrm{THz}$ polariton, $R^{(0)}=R^{(0)}(\omega)$ [red (dark gray) solid lines]. The sharp spikes in the Bragg spectrum of the acoustically driven $\mathrm{CuCl}$ crystal 


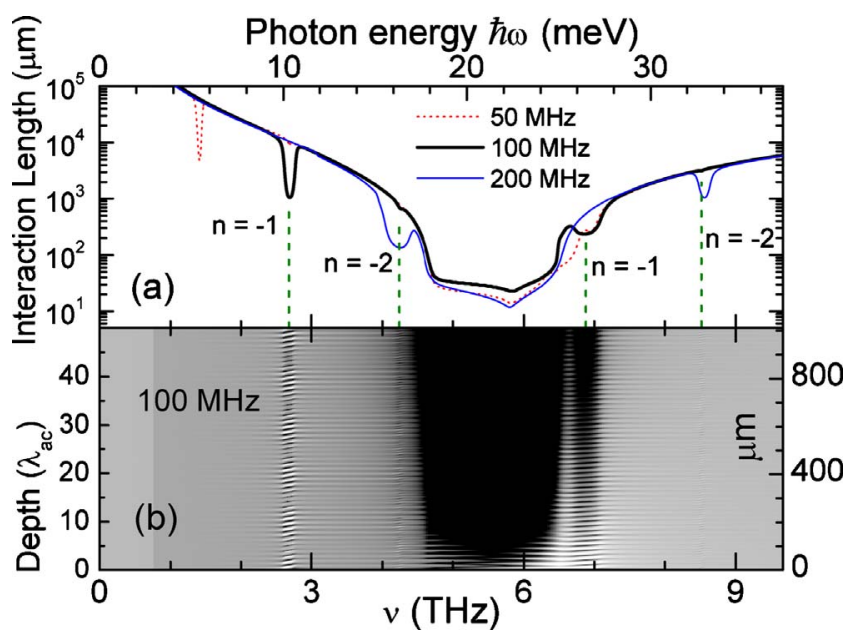

FIG. 3. (Color online) (a) The interaction length between the $\mathrm{THz}$ polariton and TA pumping wave in $\mathrm{CuCl}, \ell_{\text {int }}=\ell_{\text {int }}(\omega)$, calculated for $I_{\mathrm{ac}}=25 \mathrm{~kW} / \mathrm{cm}^{2}$ and $\nu_{\mathrm{ac}}=50,100$, and $200 \mathrm{MHz}$. (b) The electric field profile $|E(z>0, \omega)|^{2}$ evaluated for $\nu_{\mathrm{ac}}=100 \mathrm{MHz}$ $\left(\lambda_{\mathrm{ac}}=20.2 \mu \mathrm{m}\right)$. The gray-black color scale is logarithmic with black color corresponding to $E \rightarrow 0$.

are clearly seen for the one-TA-phonon and two-TA-phonon transitions, both for the upper-polariton (UP) and lowerpolariton (LP) branches [see Fig. 2(a) against Fig. 2(b)]. For $\nu_{\text {ac }} \sim 30-300 \mathrm{MHz}$, the backward scattered Bragg replica $\left|r_{-1}\right|^{2}$ peaks at the energy of the one-phonon transition and is highly efficient, with $\left|r_{-1}\right|^{2} / R \sim 50-70 \%$. The peak position and its strength are effectively tunable by changing the frequency and intensity of the AW [see Figs. 2(b)-2(d)]. This can be used for the frequency down conversion by $\omega_{\mathrm{ac}}$ $=2 \pi \nu_{\mathrm{ac}}$ of the optically induced $\mathrm{THz}$ polariton and thus of the incident light field. Generally, the backward Bragg scattering signals $\left|r_{n}\right|^{2} \propto I_{\mathrm{ac}}^{|n|}(n<0)$ peak at the spectral position of the gaps $\Delta_{N \leq \mid n}$, i.e., for the light frequency $\omega=\omega_{N}$ which satisfies the resonant Bragg condition $p_{j=0}\left(\omega_{N}\right) \simeq N k / 2$ with $N=1,2, \ldots,|n|$.

The Bragg signal $n=0$ appears as the AW-induced change in the reflectivity at incident frequency $\omega,\left|r_{0}(\omega)\right|^{2}-R^{(0)}(\omega)$. The strength of the $n=0$ signal sharply increases with decreasing detuning $\left|\omega_{N=1}-\Omega_{\mathrm{TO}}\right|$ from the TO-phonon resonance so that $\left|r_{0}\right|^{2}-R^{(0)}$ becomes dominant over $\left|r_{-1}\right|^{2}$. For $\nu_{\text {ac }} \gtrsim 1 \mathrm{GHz}$, when the one-acoustic-phonon transition within the LP branches occurs very close to $\Omega_{\mathrm{TO}}$, the AWinduced change in the reflectivity [see Fig. 2(e)] is completely determined by the $n=0$ replica and has no $\nu_{\text {ac }}$-down-converted frequency components. Thus, this operating mode can be used for TO-phonon polariton deflectors and acoustically controlled $\mathrm{THz}$ filters.

The interaction length $\ell_{\text {int }}=\ell_{\text {int }}(\omega)$ required for the formation of the Bragg signals and thus for AW control of the $\mathrm{THz}$ light field is plotted in Fig. 3(a) for various $\nu_{\mathrm{ac}}$. The sharp troughs at $\omega=\omega_{N=1}$ and $\omega_{N=2}$ in the $\ell_{\text {int }}=\ell_{\text {int }}(\omega)$ profile are due to the $n=-1$ and -2 Bragg replicas [see Fig. 3(a)]. Figure 3(b) shows the light field distribution associated with the optically induced $\mathrm{THz}$ polariton in the acoustically driven $\mathrm{CuCl}$ crystal. Apart from the broad black band [see Fig. 3(b)], which corresponds to the Restrahlen band with rather

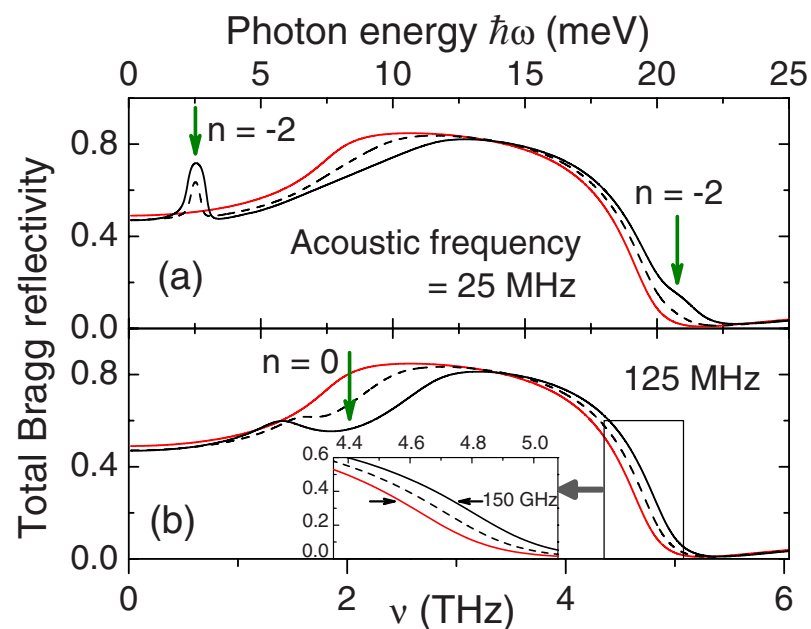

FIG. 4. (Color online) The total Bragg reflectivity $R=R(\omega)$ of a $\mathrm{TlCl}$ crystal driven by the TA wave of frequency (a) $\nu_{\mathrm{ac}}=25 \mathrm{MHz}$ and (b) $125 \mathrm{MHz}, I_{\mathrm{ac}}=100 \mathrm{~kW} / \mathrm{cm}^{2}$ (black dotted lines) and $200 \mathrm{~kW} / \mathrm{cm}^{2}$ (black solid lines). The acoustically unperturbed $\mathrm{THz}$ spectrum $R^{(0)}=R^{(0)}(\omega)$ is shown by the red (dark gray) lines. Inset: the acoustically induced Stark shift of the TO-phonon line.

weak penetration of the light field into the crystal, the narrow stripes of alternating color illustrate the formation of the Bragg replicas. The interaction length for the $n=-1$ replica at its resonant frequency $\omega=\omega_{N=1}$ is given by

$$
\ell_{\mathrm{int}}=\frac{\hbar k c^{2}}{4 m_{3} I_{\mathrm{ac}}^{1 / 2} \varepsilon_{\mathrm{b}}} \frac{\left(\omega_{N=1}^{2}-\Omega_{\mathrm{TO}}^{2}\right)^{2}}{\omega_{N=1}^{2} \Omega_{\mathrm{TO}} \Omega_{\mathrm{R}}^{2}} .
$$

Equation (10), which is valid for $\left|\Omega_{\mathrm{TO}}-\omega_{N=1}\right| \gg \gamma_{\mathrm{TO}}$ and $\ell_{\text {int }} k \gg 1$, shows the resonant decrease in $\ell_{\text {int }} \propto 1 / \sqrt{I_{\mathrm{ac}}}$ with decreasing frequency detuning from the TO-phonon resonance. In this case the interaction between the light field and pumping AW is mediated by the TO-phonon resonance, giving rise to $\ell_{\text {int }}$ of only a few tens of acoustic wavelength $\lambda_{\mathrm{ac}}$ (see Fig. 3). This is in sharp contrast with conventional acousto-optics where $\ell_{\text {int }} \sim 10^{3}-10^{4} \lambda_{\text {ac }}$ for the same operating $I_{\mathrm{ac}}$.

\section{B. Acoustically driven $\mathrm{THz}$ polaritons in bulk $\mathrm{TlCl}$}

The calculated room-temperature reflectivity $R=R(\omega)$ of a $\mathrm{TlCl}$ crystal pumped by the TA wave of frequency $\nu_{\mathrm{ac}}$ $=25 \mathrm{MHz}$ and $125 \mathrm{MHz}$ is plotted in Figs. 4(a) and 4(b), respectively. In this case, in Eqs. (1) and (3) we put $m_{3}=0$, and therefore only even-order TA-phonon-assisted transitions occur. The Bragg signals $n=-2$, due to $\left|r_{-2}\right|^{2} \propto I_{\text {ac }}^{2}$, are indicated in Fig. 4(a) by arrows, for the transitions within the LP and UP branches, respectively. Similarly to the previous case $(\mathrm{CuCl})$, for the resonant frequency $\omega_{N=2}$ close to $\Omega_{\mathrm{TO}}$ the AW-induced change in $R, \Delta R=R-R^{(0)} \sim I_{\mathrm{ac}}^{2}$ [see Fig. 4(b)], is mainly due to the $n=0$ Bragg replica. The quartic nonlinearity leads to the Stark blueshift by $2 m_{4} I_{\mathrm{ac}}$ of the TO-phonon frequency, according to Eqs. (1) and (3), as is clearly seen in Figs. 4(a) and 4(b). The Stark shift $\sim 0.1-0.2 \mathrm{THz}$ has a rather sharp contrast on the blue side of the $\mathrm{THz}$ reflectivity 
[see inset of Fig. 4(b)]. This can be used in device applications, e.g., for frequency tunable $\mathrm{THz}$ filters and deflectors.

\section{DISCUSSION}

The third- and fourth-order anharmonic nonlinearities tend to cancel each other ${ }^{17,23}$ so that the values of $m_{3}(\mathrm{CuCl})$ and $m_{4}(\mathrm{TlCl})$ we have inferred from the experimental data are in the lowest limit of their actual values. The proposed acoustic modulation of $\mathrm{THz}$ polaritons generally allow us, by comparing $n=-1$ and $n=-2$ Bragg replicas evaluated with Eqs. (2), (3), and (9), to distinguish the two nonlinearities as well as to analyze interference between the cubic and quartic anharmonic coefficients. The latter process occurs, e.g., for soft TO phonons in ferroelectric $\mathrm{LiTaO}_{3}$ and $\mathrm{LiNbO}_{3}$.

Within the used nonperturbative approach, each Bragg replica $n$ integrates all $n+s-s$ TA-phonon transitions with $s \leq n_{\max }$ : with increasing $I_{\text {ac }}$ the bare $n$-phonon transitions become dressed by higher-order processes when $n+s$ phonons are emitted and $s$ phonons absorbed. For the $n=$ -1 replica shown in Fig. 2(b), e.g., the multiphonon transitions $-1+1-1,-1+2-2$, etc., account for about $90 \%$ of $\left|r_{-1}\right|^{2}$. For $\nu_{\mathrm{ac}} \gtrsim 1 \mathrm{GHz}$ [see Fig. 2(e)], the dominant contribution to $\left|r_{0}\right|^{2}-R^{(0)}$ stems from $-s+s$ multi-TA-phonon transitions with $s>1$.

The acoustically induced modulation of far-infrared polaritons has to be particularly strong for ferroelectric soft TO phonons (e.g., in $\mathrm{LiTaO}_{3}$ and $\mathrm{LiNbO}_{3},{ }^{6}$ and bismuth titanate $^{5}$ ). In this case, a multiwell local potential for the displacive ferroelectric mode has a considerable low-wavevector component and therefore yields large values of $V_{3}$ and $V_{4}$. Very far-infrared optical phonons $(2-10 \mathrm{meV})$ in zirconium tungstate $\left(\mathrm{ZrW}_{2} \mathrm{O}_{8}\right)$ indicate anomalously high anharmonicity. ${ }^{7,8}$ The normal modes associated with soft TO phonons in this negative thermal-expansion compound are a mixture of librational and translational motion. The latter strongly couples with acoustic phonons giving rise, as we foresee, to manipulation of the $\mathrm{THz}$ polaritons by using ultrasound waves of modest $I_{\mathrm{ac}}{ }^{27}$

In accordance with Eq. (4), $m_{3}$ and $m_{4}$ scale to the sound velocity as $v_{\mathrm{s}}^{-3 / 2}$ and $v_{\mathrm{s}}^{-3}$, respectively. As a result, the use of a surface $\mathrm{AW}$ in order to control the propagation of $\mathrm{THz}$ polaritons considerably reduces the operating acoustic intensity $I_{\mathrm{ac}}: v_{\mathrm{s}}=v_{\mathrm{SAW}}$ is usually by about $20 \%$ less than $v_{\mathrm{s}}=v_{\mathrm{TA}}$.

The realistic values of the damping constant, $\hbar \gamma_{\mathrm{TO}}$ $=0.2 \mathrm{meV}$ for $\mathrm{CuCl}$ at $T=5-10 \mathrm{~K}$ (Refs. 21 and 23) and $\hbar \gamma_{\mathrm{TO}}=0.9 \mathrm{meV}$ for $\mathrm{TlCl}$ at room temperature, ${ }^{25}$ are used in our numerical simulations. Note that $\gamma_{\mathrm{TO}}$ is rather weakly temperature dependent, e.g., roughly doubling its value for $\mathrm{CuCl}$ with increasing temperature from cryogenic values to the room one. In all the materials mentioned in the paper, the polariton effect associated with TO phonons persists at room temperature. This is because the Rabi frequency of $\mathrm{THz}$ polaritons, $\hbar \Omega_{\mathrm{R}} \sim 10 \mathrm{meV}$, is much larger than the damping constant, which even for soft TO-phonon modes is usually $\hbar \gamma_{\mathrm{TO}} \sim 0.5-1 \mathrm{meV}$ only.

\section{SUMMARY}

In this paper we have proposed, theoretically described, and numerically modeled the $\mathrm{THz}$ response of far-infrared polaritons parametrically driven by a coherent acoustic field. The cubic and quartic phonon anharmonicity processes give rise to an acoustically induced Bragg grating. In this case, the TO-phonon resonance mediates and strongly enhances the acousto-optical nonlinearity leading to the anomalously small interaction length $\ell_{\text {int }} \sim 100 \mu \mathrm{m}$ between the optical and acoustic fields, which is required for the formation of the $\mathrm{THz}$ response (Bragg replicas). The main results of the work are: (i) the macroscopic Eqs. (2) and (3), which describe spatially temporal or wave-vector-frequency effective control of far-infrared polaritons and $\mathrm{THz}$ light associated with them by applying the acoustic field. (ii) The nonperturbative approach, based on Eqs. (7)-(9), to calculate the total Bragg reflectivity of TO-phonon polaritons modulated by the ultrasonic acoustic wave. (iii) The illustration of the high efficiency of the proposed effect by numerical modeling of the $\mathrm{THz}$ response of bulk $\mathrm{CuCl}$ and $\mathrm{TlCl}$ pumped by the coherent acoustic field. (iv) The large potential of the effect for device applications: the small interaction length between the $\mathrm{THz}$ light and subgigahertz acoustic field would potentially lead to a new family of $\mathrm{THz}$ microdevices, such as frequency-tunable $\mathrm{THz}$ detectors and filters, Bragg switchers, and frequency converters.

\section{ACKNOWLEDGMENTS}

We thank S. G. Tikhodeev, D. R. Khokhlov, P. Mauskopf, and R. Zimmermann for valuable discussions. This work was supported by RS (Grant No. JP0766306), EPSRC and WIMCS.

\footnotetext{
${ }^{1}$ K. Huang, Nature (London) 167, 779 (1951); Proc. R. Soc. London, Ser. A 208, 352 (1951).

${ }^{2}$ N. S. Stoyanov, D. W. Ward, T. Feurer, and K. A. Nelson, Nature Mater. 1, 95 (2002).

${ }^{3}$ T. Feurer, J. C. Vaughan, and K. A. Nelson, Science 299, 374 (2003).

${ }^{4}$ P. Y. Han and X.-C. Zhang, Meas. Sci. Technol. 12, 1747 (2001).

${ }^{5}$ S. Kojima, N. Tsumura, M. W. Takeda, and S. Nishizawa, Phys. Rev. B 67, 035102 (2003).
}

${ }^{6}$ H. J. Bakker, S. Hunsche, and H. Kurz, Rev. Mod. Phys. 70, 523 (1998).

${ }^{7}$ J. N. Hancock, C. Turpen, Z. Schlesinger, G. R. Kowach, and A. P. Ramirez, Phys. Rev. Lett. 93, 225501 (2004).

${ }^{8}$ S. L. Chaplot, Curr. Sci. 88, 347 (2005).

${ }^{9}$ A. L. Ivanov and P. B. Littlewood, Phys. Rev. Lett. 87, 136403 (2001).

${ }^{10}$ K. Cho, K. Okumoto, N. I. Nikolaev, and A. L. Ivanov, Phys. Rev. Lett. 94, 226406 (2005). 
${ }^{11}$ A. L. Ivanov, Phys. Status Solidi A 202, 2657 (2005).

${ }^{12}$ M. M. de Lima, R. Hey, P. V. Santos, and A. Cantarero, Phys. Rev. Lett. 94, 126805 (2005).

${ }^{13}$ M. M. de Lima, M. van der Poel, P. V. Santos, and J. M. Hvam, Phys. Rev. Lett. 97, 045501 (2006).

${ }^{14}$ A. L. Ivanov, in Problems of Condensed Matter Physics, edited by A. L. Ivanov and S. G. Tikhodeev (Oxford University Press, Oxford, 2008), pp. 301-322.

${ }^{15}$ V. Romero-Rochín, R. M. Koehl, C. J. Brennan, and K. A. Nelson, J. Chem. Phys. 111, 3559 (1999).

${ }^{16}$ A. A. Maradudin and A. E. Fein, Phys. Rev. 128, 2589 (1962).

${ }^{17}$ R. A. Cowley, Rep. Prog. Phys. 31, 123 (1968).

${ }^{18}$ G. P. Srivastava, The Physics of Phonons (Taylor \& Francis, New York, 1990).

${ }^{19}$ A. Debernardi, S. Baroni, and E. Molinari, Phys. Rev. Lett. 75, 1819 (1995)

${ }^{20}$ G. Deinzer, M. Schmitt, A. P. Mayer, and D. Strauch, Phys. Rev. B 69, 014304 (2004).

${ }^{21}$ C. Ulrich, A. Göbel, K. Syassen, and M. Cardona, Phys. Rev. Lett. 82, 351 (1999).

${ }^{22}$ F. Widulle, T. Ruf, A. Göbel, E. Schönherr, and M. Cardona,
Phys. Rev. Lett. 82, 5281 (1999).

${ }^{23}$ M. Cardona and T. Ruf, Solid State Commun. 117, 201 (2001).

${ }^{24}$ J. Serrano, A. Cantarero, M. Cardona, N. Garro, R. Lauck, R. E. Tallman, T. M. Ritter, and B. A. Weinstein, Phys. Rev. B 69, 014301 (2004).

${ }^{25}$ R. P. Lowndes, Phys. Rev. Lett. 27, 1134 (1971); Phys. Rev. B 6, 1490 (1972).

${ }^{26}$ G. Leibfried and W. Ludwig, in Solid State Physics, edited by F. Seitz and D. Turnbull (Academic Press, New York, 1961), Vol. 12.

${ }^{27}$ The operating acoustic intensities used in the simulations of bulk $\mathrm{CuCl}$ and $\mathrm{TlCl}$ driven by a TA wave are similar to those applied to a GaAs-based microcavity (MC) in the experiments (Refs. 12 and 13). The latter study deals with the acoustical Stark effect for MC polaritons. Our recent evaluations yield a much smaller operating intensity, $I_{\mathrm{ac}} \simeq 1-10 \mathrm{~kW} / \mathrm{cm}^{2}$, needed for the effective manipulation of TO-phonon polaritons in $\mathrm{LiNbO}_{3}$ driven by a bulk TA wave. For $\mathrm{ZrW}_{2} \mathrm{O}_{8}$, we anticipate still further reduction in the operating acoustic intensities toward a subkilowatt per square centimeter scale. 